\title{
SOBRE LA PRESENCIA DE NYCTELIA FITZROYI WATERHOUSE, 1841 Y NYCTELIA SOLIERI WATERHOUSE, 1841 (COLEOPTERA: TENEBRIONIDAE) EN LA REGIÓN DE MAGALLANES
}

\author{
ABOUT PRESENCE OF NYCTELIA FITZROYI WATERHOUSE, 1841 AND NYCTELIA SOLIERI \\ WATERHOUSE, 1841 (COLEOPTERA: TENEBRIONIDAE) IN THE MAGALLANES REGION
}

Álvaro Zúñiga-Reinoso ${ }^{1} \&$ Viviane Jerez ${ }^{1}$

\begin{abstract}
Through new records and collections review, we confirmed the presence of Nyctelia fitzroyi Waterhouse, 1841 and Nyctelia solieri Waterhouse, 1841 (Coleoptera: Tenebrionidae) in the Magallanes Region. This solves a historical confusion related with the presence or not of both species in the region. New localities are given and the biogeographic relevance is discussed.
\end{abstract}

Key words: Nyctelia fitzroyi, Nyctelia solieri, new records, Sierra Baguales.

\section{RESUMEN}

A través de nuevos registros y revisión de colecciones, se confirma la presencia de Nyctelia solieri Waterhouse, 1841 y Nyctelia fitzroyi Waterhouse, 1841 en la Región de Magallanes. Con esto se resuelve una confusión histórica relacionada con la presencia o no de ambas especies en la región, se entregan las localidades de registro y se discute sobre su relevancia biogeográfica.

Palabras clave: Nyctelia fitzroyi, Nyctelia solieri, nuevos registros, Sierra Baguales.

1 Departamento de Zoología, Facultad de Ciencias Naturales y Oceanográficas, Universidad de Concepción, Casilla 160 - C. Concepción, Chile. 


\section{ANTECEDENTES}

El género Nyctelia Latreille, 1825 (Pimelinae: Nyctelini), es un género neotropical diversificado en 64 especies, que habitan biomas áridos y semiáridos del Sur de Sudamérica, tanto de desiertos como de altamontaña (Flores 1997, Roig-Juñent \& Flores 2001, Flores \& Carrara 2006, Vidal \& Guerrero 2007). Distribuidas desde el noroeste de Argentina y Chile central, hasta el extremo sur de la Patagonia (Flores 1997, Flores \&Carrara 2006, Vidal \& Guerrero 2007, Flores 2009), la mayoría de las especies son bastante abundantes en la estepa patagónica, pudiendo encontrarse más de dos especies en simpatría (Peña 1963 a y b, Flores \& Carrara 2006). Sin embargo del punto de vista biológico, sólo existe un trabajo limitado a algunas observaciones de campo, en donde se reporta que son insectos de "hábitos diurnos, que deambulan lentamente en las planicies alimentándose principalmente de vegetación, tanto viva como muerta durante los días de sol" (Peña 1963 a y b). Por otra parte, muchas especies de Nyctelia poseen rangos de distribución poco claros, particularmente aquellas que habitan en el extremo sur de Sudamérica, como es el caso de Nyctelia fitzroyi (Waterhouse, 1841) y Nyctelia solieri (Waterhouse, 1841), citadas para la Región de Aysén e incluidas y excluidas por diversos autores de la Región de Magallanes y Antártica Chilena (Kulzer 1963, Peña 1963b, Peña 1966, Vidal \& Guerrero 2007). Es así como Kulzer (1963), cita por primera vez a N. fitzroyi para la ciudad de Punta Arenas, pero posteriormente Peña (1963b), Peña (1966) y Vidal \& Guerrero (2007), citan a esta especie solo para la Región de Aysén. Un caso parecido ocurre con $N$. solieri, registrada inicialmente por Kulzer (1963) para dos localidades de la región de Magallanes (Cerro Guido y Punta Arenas) y omitida posteriormente de ambas localidades por Peña (1963b), quien señala la presencia de $N$ solieri solo en la localidad de Cerro León en Magallanes. Posteriormente Peña (1966) en el "Catálogo de los tenebrionidos de Chile", incluye a N. solieri en la localidad Cerro Guido ya citada por Kulzer (1963), pero omite la localidad de Cerro León señalada anteriormente por Peña (1963b). Esto ha conllevado a una serie de confusiones $e$ imprecisiones en cuanto a la presencia de estas dos especies en la región.
Recientes prospecciones realizadas a la Región de Magallanes durante el año 2009 y la revisión de material conservado en algunas colecciones institucionales, resultó en el hallazgo de material de N. fitzroyi y N. solieri. Por lo que el objetivo de este trabajo es confirmar la presencia de ambas especies en la región de Magallanes, dar a conocer localidades precisas de colecta y además entregar antecedentes acerca del ambiente donde habitan y de su importancia biogeográfica. Para ello se revisó material conservado en las Colecciones de Zoología del Instituto de la Patagonia (CZIP) y Museo de Zoología de la Universidad de Concepción (UCCC). Todo el material recolectado por los autores de este trabajo será depositado en el Museo de Zoología de la Universidad de Concepción (UCCC).

Material examinado: Nyctelia fitzroyi: Chile, Provincia Última Esperanza: Estancia Sierra Leona (Lat.: 5053'16.30"S - Long: 72²1'21.70”O). 21.12.2009. leg. A. Zúñiga-Reinoso. 7 ejemplares (UCCC). det. A. Zúñiga-Reinoso. Cerro Guido. (Lat.: 5056'24.27”S - Long: 72²6’30.20”O). 17.1.1989. leg. I. Gómez. 2 ejemplares (CZIP). det. Gustavo Flores. Cerró Guido. (Lat.: 5056'24.27"S - Long: 72²6'30.20"O). 7.1.1955. leg. T. Cekalovic. 12 ejemplares (UCCC). $4 \mathrm{Km}$ al norte del puente Guido (Lat.: 5058'59.70"S- Long: 72³0'17.40”O). 21.12.2009. leg. A. Zúñiga-Reinoso. 3 ejemplares (UCCC). det. A. Zúñiga-Reinoso. Río de las Chinas (Lat.: 51 0'54.67"S - Long: 72³0'21.20"O (?)). 2.11.1952. leg. T. Cekalovic. 7 ejemplares (UCCC). (Fig. 1a).

Nyctelia solieri: Chile, Provincia Última Esperanza: Estancia Sierra Leona. (Lat.: 5053'16.30"S - Long: $\left.72^{\circ} 21^{\prime} 21.70 ” O\right)$. 21.12.2009. leg. A. ZúñigaReinoso. 3 ejemplares (CPAZ). det. A. ZúñigaReinoso. Cerro Guido (Lat.: 5056'24.27”S - Long: 72²6’30.20”O). 17.1.1989. leg. I. Gómez. 2 ejemplares (CZIP). det. Gustavo Flores.(Fig. 1b)

\section{COMENTARIOS}

Todo el material revisado y recolectado de $N$. fitzroyi y N.solieri, proviene de la Provincia de Última Esperanza y de la localidad Sierra Baguales, donde se encuentra ubicada la estancia Cerro Guido, en cuyas cercanías se encuentra el "río de las Chinas", localidad señalada en el material recolectado por Cekalovic. La localidad señalada por Kulzer (1963) 

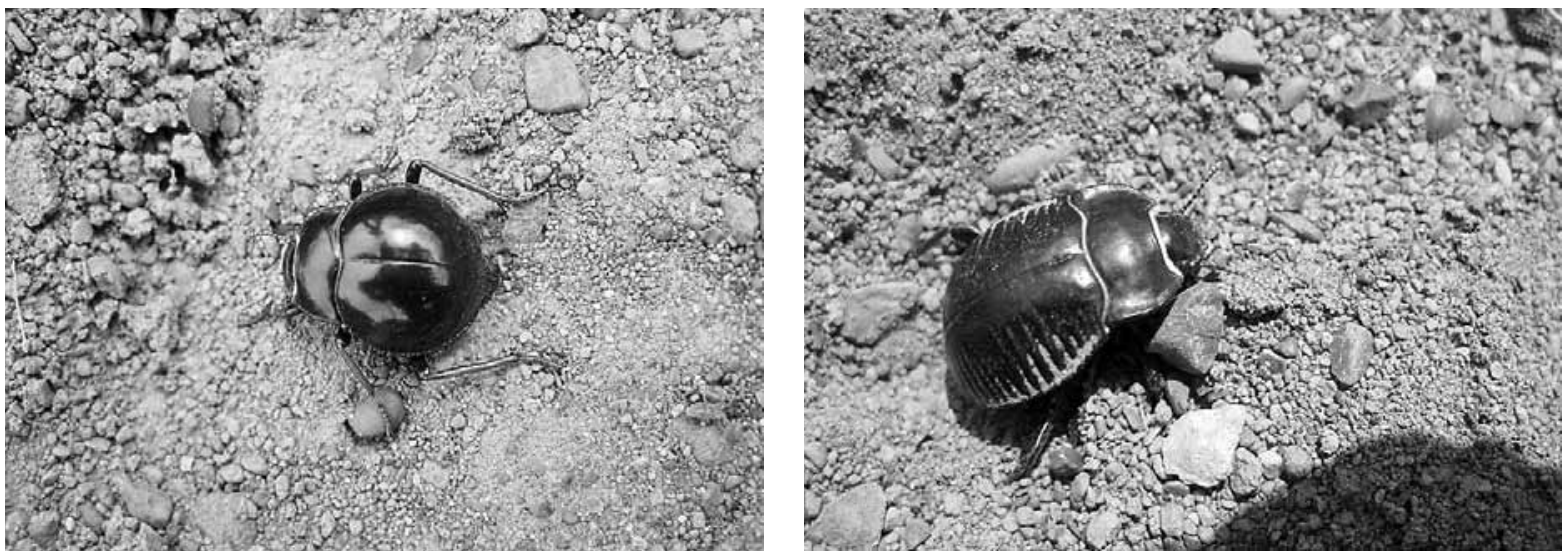

Fig. 1: a) Nyctelia fitzroyi (hembra) y b) Nyctelia solieri.(hembra). Localidad estepa de la Sierra Leona.

y Peña (1966) para N. solieri, "Sierra Leona", es coincidente con nuestras recolecciones actuales. Con respecto a la presencia de N. fitzroyi en Punta Arenas señalada por Kulzer (1963), la consideramos dudosa y probablemente por este motivo, Peña (1963, 1966) y Vidal \& Guerrero (2007), no mencionan a esta especie como parte de la fauna de la región de Magallanes. Además, la cercanía de la localidad de Cerro León entregada por Peña (1963b) con las localidades entregadas en este trabajo, hace muy probable la presencia de $N$. solieri en dicha localidad. Sin embargo, desconocemos porque Peña (1966), no volvió a citar a esta especie en esta localidad.

Biogeográficamente las localidades en las que se han registrado $N$. fitzroyi y $N$. solieri, forman parte de la estepa patagónica incluida dentro de las áreas más secas de la región y con una fuerte influencia de la estepa argentina (Pisano 1977). Esta zona, se encuentra inmersa dentro de dos regiones biogeográficas: Patagonia Subandina (Morrone et al. 2002) y Patagonia Austral (Roig-Juñent \& Flores 2001). Ambas especies constituyen componentes faunísticos de la región biogeográfica Patagonia Austral y pertenecen al área de endemismo Santacruciana (Domínguez et al. 2006) las que sobrepasan los Andes hacia la vertiente occidental de esta.

\section{AGRADECIMIENTOS}

Los autores quisieran agradecer al Proyecto DIUC N ${ }^{\circ}$ № 208.112.99 - 3. 2, por el financiamiento otorgado. Por otra parte, se agradece al Prof. Vicente Pérez y al Dr. Jorge Artigas por facilitarnos el acceso a las colecciones de CZIP y UCCC respectivamente.

\section{LITERATURA CITADA}

Dominguez, M., S. Roig-Juñent, J.J. Tassin, F. Ocampo \& G. Flores 2006. Areas of endemism of the Patagonian steppe: an approach based on insect distributional patterns using endemicity analysis. Journal of Biogeography.33: 1527-1537.

Flores, G.E. 1997. Revisión de la tribu Nycteliini (Coleoptera: Tenebrionidae). Revista de la Sociedad Entomológica Argentina. 56: 1-19. Flores, G. \& R. Carrara 2006. Two new species of Nyctelia Latreille (Coleoptera: Tenebrionidae) from western Argentinean high mountains. AnnalesZoologici. 56: 487-495.

Flores G.E. 2009. Revision of some types of the South American tribes Nycteliini, Praocini, and Scotobiini (Coleoptera: Tenebrionidae), with new synonymies. Zootaxa 1985: 21- 33.

Kulzer, H. 1963. Revision der südamerikanischen Gattung Nyctelia Latr. (Col. Teneb.) (24 Beitrag zur Kenntnis der Tenebrioniden). Entomologische Arbeiten aus dem Museum George Frey. 14: 1-171.

Morrone, J.J., S. Roig-Juñent \& G.E. Flores 2002. Delimitation of biogeographic districts in central Patagonia(southern South America), based on beetle distributional patterns (Coleoptera: Carabidae and Tenebrionidae). Revista del Museo Argentino de Ciencias Naturales. 4: 1-6

Peña, L. 1963a. Las Nyctelia (Coleoptera, Tenebrionidae). Entomologische Arbeiten aus dem Museum George Frey. 9:72-75. 
Peña, L. 1963b. Las Nyctelia (Coleoptera, Tenebrionidae). Noticiario Mensual del Museo Nacional de Historia Natural. 86:6-8.

Peña, L. 1966, Catálogo de los Tenebrionidae (Coleoptera) de Chile. Entomologische Arbeitenausdem Museum George Frey. 17: 397-453.

Roig-Juñent, S. \& G. Flores 2001. Historia biogeográfica de las áreas áridas de América del sur austral. En: Llorente, J. \& J. Morrone (eds.). Introducción a la biogeografía en Latinoamérica: Teorías, conceptos, métodos y aplicaciones. Las Prensas de Ciencias, Facultad de Ciencias, UNAM. México. 277 pp.

Vidal, P. \& M. Guerrero 2007. Los Tenebriónidos de Chile. 1a edición. Ediciones Universidad Católica de Chile. Santiago, Chile. 478 pp. 